Pacific Journal of Mathematics

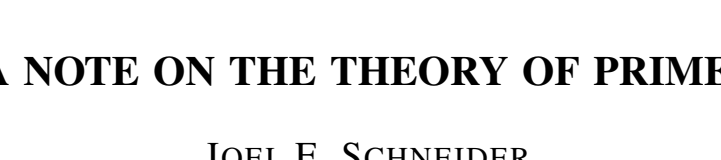




\title{
A NOTE ON THE THEORY OF PRIMES
}

\author{
J. E. SCHNEIDER
}

In this paper we find those commutative rings for which the theory of primes is subsumed under classical ideal theory, that is, for which every finite prime is an ideal. The characterization is given in terms of domains with this property and they are shown to form a class of domains from number theory. In addition we give two characterizations of the primes of a subring of a global field. The first establishes them as the nontrivial preprimes whose complements are multiplicatively closed and the second relates the space of all primes to that of the quotient field.

The concept of a prime for commutative rings with identity was introduced by Harrison in 1966 .

In what follows all rings are commutative and have a unity and all primes are finite. $X(R)$ denotes the set of primes of a ring $R$ and $X^{\prime}(R)$ denotes the set of valuation preprimes (preprimes $T$ such that for each finite $E \subset R, T \cap E=\varnothing \Rightarrow$ there is $P \in X(R)$ with $T \subset P$ and $P \cap E=\varnothing)$. For a preprime $T$ of $R$ which is closed under subtraction, define the idealizer $A(T)$ of $T$ in $R$ by $A(T)=\{a \in R: a T \subset T\} . A(T)$ is a subring of $R$ in which $T$ is an ideal.

1. Call a ring a $C$-ring if every finite prime of it is an ideal. It is easy to check that the class of $C$-rings is closed under taking subrings and homomorphic images.

THEOREM 1. The following are equivalent for a ring $R$ :

(1) $R$ is a C-ring;

(2) $X(R)=\{$ maximal ideals of $R\}$;

(3) $R / P$ is a $C$-ring, for each minimal prime ideal $P$ of $R$;

(4) $X^{\prime}(R)=\operatorname{Spec}(R)$.

Proof. That $(4) \Rightarrow(2) \Rightarrow(1) \Rightarrow(3) \quad$ is clear. In any case, $\operatorname{Spec}(R) \subset X^{\prime}(R)$ [1, Lemma 2.6]. Let $P \in X^{\prime}(R) . \quad P$ contains a minimal prime ideal $Q$ of $R$ and $P / Q \in X^{\prime}(R / Q)$. Then $P / Q$ is the intersection of the primes of $R / Q$ which contain it; so, if $R / Q$ is a $C$-ring, then $P / Q \in \operatorname{Spec}(R / Q)$ and $P \in \operatorname{Spec}(R)$.

Because of condition (3), we turn to the classification of $C$-domains. If $S$ denotes the ring of rational integers or a ring of polynomials in one variable over a finite field, then one checks that the polynomial 
ring $S[X]$ has primes which are not ideals. Thus, since a subring of a $C$-ring is also a $C$-ring, the transcendence degree of a $C$-domain is zero or one, the latter only if it has nonzero characteristic.

Let $R$ denote a domain whose quotient field $F$ is absolutely algebraic and has characteristic zero. Let $R^{\prime}$ denote the integral closure of $R$ and $S^{\prime}$ the integral closure in $F$ of its prime subring. Let $S=R \cap S^{\prime} . \quad S^{\prime}$ (and hence $S$ ) is a $C$-domain and $P \rightarrow P \cap S^{\prime}$ gives a bijection from $X(F)$ onto $X\left(S^{\prime}\right)$. In fact, the finite dimentional case follows from Proposition 3.4 of [1] and the general case follows immediately from it. Since for $P \in X(F), P \cap R^{\prime}$ is an ideal of $R^{\prime}$ if and only if $R^{\prime} \subset A(P)$, we have that $R^{\prime}$ is a $C$-domain if and only if $R^{\prime}=S^{\prime}$. Now $S^{\prime} \subset R^{\prime} \subset F$ yields that $P \rightarrow P \cap S^{\prime}$ gives a bijection from $X\left(R^{\prime}\right)$ onto $X\left(S^{\prime}\right)$ and $P \rightarrow P \cap R^{\prime}$ gives a bijection from $X(F)$ onto $X\left(R^{\prime}\right)$. Since $S \subset S^{\prime}$ are both $C$-domains, $P \cap S \in X(S)$ for any $P \in X\left(R^{\prime}\right)$.

\section{Lemma 2. If $P \in X(F)$, then $P \cap R \in X(R)$.}

Proof. Note that it suffices to consider the case where $F$ is finite dimensional over its prime subfield. Moreover, since $P \cap R^{\prime} \in X\left(R^{\prime}\right)$ for any $P \in X(F)$, as already noted, it suffices to show that $P \cap R \in X(R)$ for $P \in X\left(R^{\prime}\right)$. We may assume that $R \neq R^{\prime}$, whence $S \neq S^{\prime}$. Let $c(S)($ resp. $c(R))$ denote the conductor of $S$ in $S^{\prime}\left(\operatorname{resp} . R\right.$ in $\left.R^{\prime}\right)$. Recall that $(0) \neq c(S) \subset c(R)$. Now let $P \in X\left(R^{\prime}\right)$ and just suppose that $P \cap R$ is not in $X(R)$. Then $P \cap R \subset T \in X(R)$, since $P \cap R$ is a preprime of $R$. But $T$ is a preprime of $R^{\prime}$, so $T \subset Q \in X\left(R^{\prime}\right)$. Then $P \cap R \subset T=Q \cap R$. Since $P \cap R \neq T$, we have $P \neq Q$ and $P \cap S^{\prime} \neq Q \cap S^{\prime}$. Now $P \cap S=P \cap R \cap S \subset Q \cap R \cap S=Q \cap S$. But $P \cap S^{\prime} \in X\left(S^{\prime}\right)$ yields $P \cap S=\left(P \cap S^{\prime}\right) \cap S \in X(S)$. Thus $P \cap S=Q \cap S$. Hence, by [2, p. 91], $c(S) \subset P \cap Q$. By the approximation theorem, there is $a \in F$ whose (normalized exponential) value is -1 at $P,+1$ at $Q$, and nonnegative otherwise. Note that $a$ is in $R^{\prime}$. Let $0 \neq b \in c(S) \subset P \cap Q$ and let $n$ be its (positive) value at $P$. Then $b a^{n} \in Q \cap S^{\prime}$. Also $b a^{n} \in c(S) R^{\prime} \subset c(R) R^{\prime} \subset R$. Thus $b a^{n} \in Q \cap S^{\prime} \cap R=$ $Q \cap S=P \cap S$. But this a contradiction, since $b a^{n}$ was constructed to have value zero at $P$.

Now assume that $R$ is a $C$-domain. We will show that then $R^{\prime}$ is a $C$-domain, so $R^{\prime}=S^{\prime}$ and $R$ is absolutely integral. Let $P \in X\left(R^{\prime}\right)$. We must show that $A(P)=R^{\prime}$. If $R=R^{\prime}$ we are done, so we assume that $R \neq R^{\prime}$. By the lemma, $P \cap R \in X(R)$. But then $A(P) \cap R=A(P \cap R)=R$, so $R \subset A(P)$. Since $A(P)$ is integrally closed in $R^{\prime}$, it is integrally closed in $F$. Then $R \subset A(P)$ yields $R^{\prime} \subset A(P)$, whence $A(P)=R^{\prime}$. 
We have shown that a domain of characteristic zero is a $C$-domain if and only if it is absolutely integral. An absolutely integral domain of nonzero characteristic is always a $C$-domain (such a domain has a unique prime and a unique proper ideal, namely (0)). We are faced with determining which domains of nonzero characteristic and transcendence degree one are $C$-domains. This case is handled in a fashion similar to the above case with the usual care necessary to handle a finite set of primes (those arising from the "infinite" valuations of the quotient field). If $R$ is such a domain, $F$ its quotient field, and $R^{\prime}$ its integral closure, then $P \rightarrow P \cap R^{\prime}$ gives a bijection from $X(F)$ onto $X\left(R^{\prime}\right)$ or onto $X\left(R^{\prime}\right) \cup\{(0)\}$, depending on whether there is not or is a unique valuation ring of $F$ which does not contain $R$. For $P \in X(F), P \cap R \in X(R)$ unless there is a unique valuation ring of $F$ which does not contain $R$ and $P$ is its maximal ideal, in which case $P \cap R=(0)$. Now if $R$ is a $C$-domain, then so is $R^{\prime}$. But then $R^{\prime}$ (or equivalently $R$ ) is contained in all but one valuation ring of $F$. We omit the details. This completes the classification of $C$-domains.

THEOREM 3. $R$ is a $C$-domain if and only if $R$ is absolutely integral or $R$ has nonzero characteristic, has transcendence degree one, and is contained in all but one valuation ring of its quotient field.

REMARKs. The hypothesis on the transcendence degree of $R$ is superfluous in Theorem 3 , but we admit it to emphasize that $C$-domains lie in the realm of number theory. Since the Krull dimension of a $C$-domain is zero or one, the same is true for $C$-rings. Using Proposition 2.11 of [1], it is easy to check that $X(R)=\operatorname{Spec}(R)$ if and only if $R / \sqrt{0}$ is a generalized Boolean ring. Theorem 1 answers a question arising in the theory of valuations of commutative rings introduced by Manis [3], namely, every valuation of a commutative ring is trivial if and only if it is a $C$-ring.

2. Call a domain a global ring if its quotient field is a global field, that is, a finitely generated field of adjusted transcendence degree one (equals transcendence degree plus one or zero depending on whether the characteristic is zero or not). We seek to characterize the primes of a global ring. Since the infinite primes are easily seen to arise in the same way and correspond exactly to the infinite primes of its quotient field [1, Propositions 3.5 and 3.6], we will consider only finite primes.

Let $R$ denote any domain of adjusted transcendence degree one 
and $F$ its quotient field. Let $T$ denote a nonzero preprime of $R$ whose complement in $R$ is multiplicatively closed. Then $T$ is closed under subtraction, $A=A(T)$ also has adjusted transcendence degree one, and the complement of $T$ in $A$ is multiplicatively closed. Thus $T$ is a maximal ideal of $A$ and a (finite) prime of $A$. We seek to show that $T$ is a prime of $R$. We may assume that $A \neq R$, i.e., that $T$ is not an ideal of $R$. Let $S$ and $B$ denote the integral closures in $F$ of $R$ and $A$ respectively. Then $B \subset S, R \cap B=A$, and $B \neq S$.

Let $E(R)$ denote the set of valuation rings of $F$ which do not contain $R$. Since $B \subset S$ and $B \neq S, E(R)$ is not empty. It suffices to consider the case where $R$ is a global ring and $E(R)$ is finite. In fact, assume that $T$ is properly contained in a prime $P$ of $R$. Let $a \in P, a \notin T$, and $0 \neq b \in T$. Let $R_{1}$ denote the subring of $R$ generated by $a$ and $b$. Then $R_{1}$ is a global ring (otherwise $a$ is a root of unity and $1 \in P) . E\left(R_{1}\right)$ is the set of valuation rings of the quotient field of $R_{1}$ which exclude $a$ or $b$, so $E\left(R_{1}\right)$ is finite. $T \cap R_{1}$ is a nonzero preprime of $R_{1}$ whose complement in $R_{1}$ is multiplicatively closed, and $T \cap R_{1}$ is properly contained in the preprime $P \cap R_{1}$.

We omit the proof of the following lemma since it is a strightforward application of the approximation theorem and the fact that the conductor of a global ring in its integral closure is not zero.

Lemma 4. Let $L$ denote a global ring and $V_{1}, \cdots, V_{n}$ a set of valuation rings of the quotient field of $L$ which do not contain $L$. Then $L \cap V_{1} \cap \cdots \cap V_{n}$ is an irredundant intersection.

Let $M=M(T, R)=\{V \in E(R): A \subset V\}$. Since $B \neq S, M$ is not empty. Let $M=\left\{V_{1}, \cdots, V_{n}\right\}$. Then $B=S \cap V_{1} \cap \cdots \cap V_{n}$ and $A=R \cap V_{1} \cap \cdots \cap V_{n}$. Let $P_{i}$ denote the maximal ideal of $V_{i} \in M$ and let $N=\left\{P_{1}, \cdots, P_{n}\right\}$. Recall that $N \subset X(F)$.

Lemma 5. $T=A \cap P$, for $P \in N$.

Proof. Just suppose that $T \neq A \cap P$, for all $P \in N$. Let $Q \in X(F)$ with $T \subset Q$. Since $T=A \cap Q$ and $T$ is an ideal and a prime of $A, A \subset A(Q) . \quad Q \notin N$, so $A(Q) \notin E(R)$. Thus $R \subset A(Q)$ and $R \cap Q$ is a maximal ideal of $R$. Since $T$ is not an ideal of $R, R \cap Q \neq T=A \cap Q$. Let $b \in R \cap Q$ with $b \notin A \cap Q$. Now, for $P_{i} \in N, A \cap P_{i}$ is a maximal ideal of $A$ and since $A \cap P_{i} \neq T=A \cap Q$, there is $a_{i} \in A \cap P_{i}$ with $a_{i} \notin A \cap Q$. Let $a=a_{1} a_{1} \cdots a_{n}$. Let $w$ (resp. $v_{i}$ ) denote the normalized exponential valuation of $F$ associated with $Q$ (resp. $P_{i}$ ). Then $w(b)>0, w(a)=0$, and $v_{i}(a)>0$. We fix $m>0$ so that $v_{i}\left(a^{m} b\right)>0$, for $1 \leqq i \leqq n$. Then $a^{m} b \in R \cap V_{1} \cap \cdots \cap V_{n} \cap Q=A \cap Q=T$. But 
$a, b \in R$, so $a^{m} b \in T$ implies that $a \in T$ or $b \in T$. By this contradiction $T=A \cap P$, for some $P \in N$.

Now reindex $N$ if necessary so that $T=A \cap P_{i}$ for $1 \leqq i \leqq s$, and $T \neq A \cap P_{i}$ for $s<i \leqq n$. Just suppose $s<n$. Let $R_{1}=R \cap$ $V_{1} \cap \cdots \cap V_{s} . \quad T$ is a preprime of $R_{1}$ with multiplicatively closed complement in $R_{1}$. By Lemma $4, A \neq R_{1}$ and $M\left(T, R_{1}\right)=\left\{V_{s+1}, \cdots, V_{n}\right\}$. Applying the first part of this proof to $T$ and $R_{1}$, we get $T=R_{1} \cap P_{i}$, for some $s<i \leqq n$. But then $T=T \cap R=R \cap P_{i}$, a contradiction.

Lemma 6. Let $L$ denote a global ring, $T$ a nonzero preprime of $L$ with a multiplicatively closed complement in $L$, and $A$ the idealizer of $T$ in $L$. Assume that $E(L)$ is finite and that $M(T, L)=\{V\}$. Then $T \in X(L)$.

Proof. Let $P$ denote the maximal ideal of $V$. Then Lemma 5 yields $\quad T=A \cap P . \quad$ But $M(T, L)=\{V\}$ yields $A=L \cap V . \quad$ Thus $T=L \cap V \cap P=L \cap P$. But if $L$ has characteristic zero, Lemma 2 yields $T=L \cap P \in X(L)$. If $L$ has nonzero characteristic, we consider $E(L)$. At least $V \in E(L)$. If $E(L)=\{V\}$, then $A=L \cap V$ is a finite field and $T=(0)$. Hence $E(L)$ is not a singleton and $T=L \cap P \in X(L)$.

We can now give the first characterization.

Proposition 7. Let $R$ be a domain of adjusted transcendence degree one. A necessary and sufficient condition that a nonzero preprime of $R$ be a prime is that its complement be multiplicatively closed.

Proof. Necessity holds for arbitrary commutative rings. [1, Proposition 2.1]. To prove the sufficiency, we have already noted that we may assume that $R$ is a global ring, that $E(R)$ is finite, and that the idealizer $A$ of $T$ in $R$ is not $R$. In the notation established above, $M(T, R)=\left\{V_{1} \cdots, V_{n}\right\}$. By Lemma 6 , we need only show that $n=1$. Just suppose $n \geqq 2$. Let $L=R \cap V_{3} \cap \cdots \cap V_{n}$. Then $A=$ $L \cap V_{1} \cap V_{2}$. Let $R_{1}=L \cap V_{1}$, so $A=R_{1} \cap V_{2}$. By Lemma 6 applied to $R_{1}$ and $T$, we have that $T \in X\left(R_{1}\right)$; and by Lemma $5, T=$ $A \cap P_{1} \subset R_{1} \cap P_{1}$, so $T=P_{1} \cap R_{1}$. Then, since $A$ is the idealizer of $T$ in $R_{1}, A=V_{1} \cap R_{1}=R_{1}$, in contradiction to Lemma 4 .

For a commutative ring $R, X(R)$ is topologized by taking the sets $U(a)=\{P \in X(R): a \notin P\}$, for all $a \in R$, as subbasic open sets. Let $R$ denote a global ring and $F$ its quotient field. We fix an element $x \in R$ so that $x$ is not absolutely algebraic, if $\operatorname{char}(R) \neq 0$, and $x=1$, otherwise. Let $F_{0}$ (resp. $R_{0}$ ) denote the subfield (resp. subring) of $F$ generated by $x$. Let $V=U(1 / x) \subset X(F)$. Let $S$ (resp $S_{0}$ ) denote the 
integral closure of $R$ (resp. $R_{0}$ ) in $F$. We fix $c \in R_{0}$ with $c S \subset R$ and $c \neq 0$. Note that for $P \in V, x \in A(P)$; so $c \in R_{0} \subset A(P)$.

For commutative rings $K \subset L$, let $\pi(L / K)$ denote the map from $X(L)$ to the power set of $K$ given by $\pi(L / K)(P)=P \cap K$. We have noted that $\pi\left(F / S_{0}\right)$ restricted to $V$ is a bijection onto the set of maximal ideals of $S_{0}$, and $\pi\left(F_{0} / R_{0}\right)$ restricted to $\left\{P \in X\left(F_{0}\right): 1 / x \notin P\right\}$ is a bijection onto $X\left(R_{0}\right)$ [cf. 1, Proposition $3.4 \mathrm{ff}$.]. Thus $S_{0} \subset S \subset F$ implies that $\pi(F / S)$ restricted to $V$ is injective and that for $P \in V$, $\pi(F / S)(P) \neq(0)$. Then by proposition $7, \pi(F / S)(V) \subset X(S)$. Note that, for $P, Q \in X(F)$ with $P \cap R=Q \cap R$, either both $P$ and $Q$ are in $V$ or both are not. In fact, $P \in V$ if and only if $P \cap R_{0} \neq(0)$.

Lemma 8. Let $P, Q \in V$. If $P \neq Q$ and $P \cap R=Q \cap R$, then $c \in P \cap Q$.

Proof. Let $T=(P \cap S)+(Q \cap S)+(P \cap S)(Q \cap S) . \quad T$ is closed under addition and multiplication, but $T$ properly contains $P \cap S \in X(S)$. Thus $T$ is not a preprime of $S$ and hence $1 \in T$. Then $c \in c T \subset P \cap Q$.

Since $X(F)$ is cofinite space, $C=\{P \in X(F): 1 / x \in P$ or $c \in P\}$ is finite. By Lemma 8, $\pi(F / R)$ restricted to the complement $C^{\prime}$ of $C$ is injective. $X(R)$ lies in the range of $\pi(F / R)$, and we may choose a subset $D$ of $C$ on which $\pi(F / R)$ is injective and so that $\pi(F / R)(D)=$ $\pi(F / R)(C)$. Then $D \cup C^{\prime}$ is a cofinite set and an open set, and $\pi(F / R)$ restricted to $D \cup C^{\prime}$ is a bijection onto $X(R)$. Since $X(F)$ and $X(R)$ are cofinite spaces, this map is a homeomorphism. We have proven.

Proposition 9. Let $R$ denote a global ring and $F$ its quotient field. Then $X(R)$ is homeomorphic to an open (cofinite) subset of $X(F)$ and the homeomorphism is induced by $\pi(F / R)$.

\section{REFERENCES}

1. D. K. Harrison, Finite and infinite primes for rings and fields, Memoirs Amer. Math. Soc. 68 (1966).

2. W. Krull, Idealtheorie, Springer, Berlin, 1935.

3. M. E. Manis, Extension of valuation theory, announced in Bull. Amer. Math. Soc. 73 (1967), p. 735, and to appear.

Received December 1968. This research was completed while the author held an NDEA Fellowship at the University of Oregon.

The Pennsylvania State University

University Park, Pennsylvania 


\section{PACIFIC JOURNAL OF MATHEMATICS}

\section{EDITORS}

H. ROYDEN

Stanford University

Stanford, California

\section{RichaRd PIERCE}

University of Washington Seattle, Washington 98105
J. DugundJI

Department of Mathematics

University of Southern California

Los Angeles, California 90007

BASIL GORDON

University of California

Los Angeles, California 90024

\section{ASSOCIATE EDITORS}
E. F. BECKENBACH
B. H. NeUMANN
F. WOLF
K. YOSHIDA

\section{SUPPORTING INSTITUTIONS}

UNIVERSITY OF BRITISH COLUMBIA CALIFORNIA INSTITUTE OF TECHNOLOGY

UNIVERSITY OF CALIFORNIA

MONTANA STATE UNIVERSITY

UNIVERSITY OF NEVADA

NEW MEXICO STATE UNIVERSITY

OREGON STATE UNIVERSITY

UNIVERSITY OF OREGON

OSAKA UNIVERSITY

UNIVERSITY OF SOUTHERN CALIFORNIA

\author{
STANFORD UNIVERSITY \\ UNIVERSITY OF TOKYO \\ UNIVERSITY OF UTAH \\ WASHINGTON STATE UNIVERSITY \\ UNIVERSITY OF WASHINGTON \\ AMERICAN MATHEMATICAL SOCIETY \\ CHEVRON RESEARCH CORPORATION \\ TRW SYSTEMS \\ NAVAL WEAPONS CENTER
}

The Supporting Institutions listed above contribute to the cost of publication of this Journal, but they are not owners or publishers and have no responsibility for its content or policies.

Mathematical papers intended for publication in the Pacific Journal of Mathematics should be in typed form or offset-reproduced, double spaced with large margins. Underline Greek letters in red, German in green, and script in blue. The first paragraph or two must be capable of being used separately as a synopsis of the entire paper. It should not contain references to the bibliography. Manuscripts, in duplicate if possible, may be sent to any one of the four editors. Please classify according to the scheme of Math. Rev. 36, 1539-1546. All other communications to the editors should be addressed to the managing editor, Richard Arens, University of California, Los Angeles, California, 90024.

50 reprints are provided free for each article; additional copies may be obtained at cost in multiples of 50 .

The Pacific Journal of Mathematics is published monthly. Effective with Volume 16 the price per volume (3 numbers) is $\$ 8.00$; single issues, $\$ 3.00$. Special price for current issues to individual faculty members of supporting institutions and to individual members of the American Mathematical Society: $\$ 4.00$ per volume; single issues $\$ 1.50$. Back numbers are available.

Subscriptions, orders for back numbers, and changes of address should be sent to Pacific Journal of Mathematics, 103 Highland Boulevard, Berkeley, California, 94708.

PUBLISHED BY PACIFIC JOURNAL OF MATHEMATICS, A NON-PROFIT CORPORATION

Printed at Kokusai Bunken Insatsusha (International Academic Printing Co., Ltd.), 7-17. Fujimi 2-chome, Chiyoda-ku, Tokyo, Japan. 


\section{Pacific Journal of Mathematics}

\section{Vol. 30, No. $3 \quad$ November, 1969}

Willard Ellis Baxter, Topological rings with property $(Y) \ldots \ldots \ldots \ldots . \ldots 5$

Sterling K. Berberian, Note on some spectral inequalities of $C . R$.

Putnam ..................................... 573

David Theodore Brown, Galois theory for Banach algebras . ........... 577

Dennis K. Burke and R. A. Stoltenberg, A note on p-spaces and Moore spaces ........................................ 601

Rafael Van Severen Chacon and Stephen Allan McGrath, Estimates of positive contractions....................................... 609

Rene Felix Dennemeyer, Conjugate surfaces for multiple integral problems in the calculus of variations ........................... 621

Edwin O. Elliott, Measures on countable product spaces.............. 639

John Moss Grover, Covering groups of groups of Lie type .............. 645

Charles Lemuel Hagopian, Concerning semi-local-connectedness and cutting in nonlocally connected continua .................. 657

Velmer B. Headley, A monotonicity principle for eigenvalues ........... 663

John Joseph Hutchinson, Intrinsic extensions of rings . . . . . . . . . . . . . 669

Harold H. Johnson, Determination of hyperbolicity by partial

prolongations .................................. 679

Tilla Weinstein, Holomorphic quadratic differentials on surfaces in $E^{3} \ldots 697$

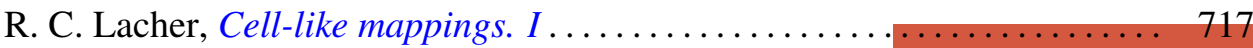

Roger McCann, A classification of centers

Curtis L. Outlaw, Mean value iteration of nonexpansive mappings in a

Banach space...

Allan C. Peterson, Distribution of zeros of solutions of a fourth order

differential equation.

Bhalchandra B. Phadke, Polyhedron inequality and strict convexity .. 765 Jack Wyndall Rogers Jr., On universal tree-like continua .

Edgar Andrews Rutter, Two characterizations of quasi-Frobenius rings

G. Sankaranarayanan and C. Suyambulingom, Some renewal theorems concerning a sequence of correlated random variables...

Joel E. Schneider, A note on the theory of primes........ . .

Richard Peter Stanley, Zero square rings .................

Edward D. Tymchatyn, The 2-cell as a partially ordered space

Craig A. Wood, On general Z.P.I.-rings................ 